\title{
The Indefiniteness and Focusing of Question Words
}

\author{
Andreas Haida \\ Humboldt University, Berlin
}

\section{Introduction}

Question words show striking similarities among the languages of the world: They are typically morphologically related to indefinites and they are typically, if not universally, focused. ${ }^{1}$ These two properties have been semantically accounted for in various ways, but so far they have not been integrated into a single coherent theory. I will show that an integration can be achieved by combining two independently motivated accounts, which up to now have not been considered together. The analysis arrived at will be shown to yield a number of answerhood conditions that are left unexplained by the pertinent semantic theories of questions and to explain intervention effects in $w h$-questions.

\section{Some Facts about Question Words}

\subsection{Question Words are Closely Related to Indefinites}

Indefinite and interrogative pronouns are typically closely related in form (Haspelmath 1997, Bhat 2000). ${ }^{2}$ Among the languages that show this relationship, we can identify two major classes: In the first class, indefinite and interrogative pronouns are identical in form. ${ }^{3}$ In the second class, indefinite pronouns are derived from interrogative pronouns. I will concentrate on the first class, because I consider the identity of indefinite and interrogative pronouns as the basic phenomenon. ${ }^{4}$

For an illustration of the indefinite-interrogative affinity, consider the example in (1), which is a string of Lakhota words. This string has two readings, a yes/no-question and a $w h$-question reading (see the paraphrases in (1a) and (b), respectively).

I am grateful to the audience of SALT XVIII for their helpful comments. I owe special thanks to Adrian Brasoveanu, Paul Elbourne, and Anna Szabolcsi for giving me ideas for future research.

${ }^{1}$ In addition to this, question words usually form a morphologically distinct class, for example, the class of $w h$-words in English. For reasons of space, I will not discuss this property.

${ }^{2}$ Here and below, I use "pronoun" in the broad sense of Haspelmath (1997), including proadverbs and determiners.

${ }^{3}$ To be precise, the formal identity holds except for the focus feature borne by the interrogative occurrences of these pronouns. See section 2.2 for discussion.

${ }^{4}$ See Kratzer and Shimoyama (2002) for an analysis that takes the derivational relationship between various pronoun paradigms as starting point. See Haida (2007) for a thorough comparison of this approach with the dynamic-semantic approach to be presented here. 
(1) šúka ki táku yaxtáka he

dog the something/what bit Q

a. 'Did the dog bite something?'

b. 'What did the dog bite?'

Note that on the first reading, the pronoun táku functions as an indefinite, and on the second as a question word.

Observations like this ${ }^{5}$ suggest that interrogative pronouns have the same denotation as the corresponding indefinite pronouns. Therefore, I assume that $w h$ phrases denote existential generalized quantifiers (cf. Karttunen 1977). In this paper, I use a provisional translation procedure between natural-language expressions and expressions of a logical calculus to avoid confusion when discussing the meaning of the latter (see section 3). ${ }^{6}$ Thus, for example, the $w h$-pronoun $w h o_{n}$ is given the following translation (where $n$ is a numerical index). ${ }^{7}$

$$
\mathrm{who}_{n} \leadsto \lambda P . \exists u_{n} . P(i)\left(u_{n}\right)
$$

As will be argued in section 2.3, the existential quantifier in (2) must be a dynamic quantifier. Hence, the term in (2) and the logical expressions discussed in the following sections are expressions of a dynamic type logic. This logic will be specified in section 3.2 and 3.3.

\subsection{Question Words are Focused}

There is more to be said about the ambiguity of (1). According to Van Valin (1993: 98), the two readings are disambiguated by the location of the focus: (1) is interpreted as a constituent question if and only if the focus falls on the pronoun táku. Put differently, táku functions as a question word if and only if it is focused. Here, being "focused" is to be understood, first of all, in the formal sense of the term, but I will argue in a minute that question words are focused in a semantic sense too.

The focusing of question words has been observed in a large variety of languages, and it is also recognized that this phenomenon is very widespread or even universal (see, for example, É. Kiss 1995: 23). However, so far little attention has been paid to the question of what interpretation the focus feature borne by question words receives (for a notable exception, see Beck 2006). To approach this question, let us consider the syntactic distribution of question words in Hungarian. The paradigm in (3) shows that question contituents must occur in the immediate preverbal position, which is the focus position in Hungarian (É. Kiss 1998: 249). ${ }^{8}$

\footnotetext{
${ }^{5}$ The phenomenon under consideration can also be observed in German (Zaefferer 1991), Korean (Choe 1995), and a number of other languages (Haspelmath 1997).

${ }^{6}$ Once the relevant semantic objects are established, natural-language expressions (viz. LF structures) should be directly related to their denotations.

${ }^{7}$ I assume for simplicity that $w h$-pronouns do not have a restriction.

${ }^{8}$ This does not hold for miért 'why', for reasons that are unclear to me. Also see section 4.2 for the syntax multiple questions in Hungarian.
} 
a. A huzat [melyik szoba ablakait] törte be? the draft which room's windows.ACC broke in

'The windows of which room did the draft break?'

b. [Melyik szoba ablakait] törte be a huzat?

c. * [Melyik szoba ablakait] a huzat törte be?

d. * [Melyik szoba ablakait] be törte a huzat?

e. * A huzat [melyik szoba ablakait] be törte?

I propose to take the distributional facts of Hungarian at face value. In Szabolcsi (1981), it is shown that preverbal focus constituents receive an exhaustive interpretation. Let us assume that this also holds for question constituents. I propose that exhaustification is achieved by the semantics of a specific focus feature $F_{e}$, and that in Hungarian, this feature must be syntactically licensed in the preverbal focus position. Following Szabolcsi (1994), I assume that the $F_{\mathrm{e}}$ feature translates into the term given in (4).

$$
\mathrm{F}_{\mathrm{e}} \leadsto \lambda \mathscr{Q} \lambda P . \mathscr{Q}(i)\left(\lambda i \lambda v^{\prime}\left(v^{\prime}=\sigma v \cdot P(i)(v)\right)\right)
$$

The operator in (4) turns a generalized quantifier into the corresponding exhaustive generalized quantifier. This is achieved by means of the $\sigma$-operator, which is defined slightly differently here than in Link (1983), namely as described in (5). ${ }^{9}, 10$

$$
\begin{aligned}
& \sigma v . P(i)(v) \text { is the maximal sum individual } \\
& \text { that satisfies the plural predicate of } \lambda v . P(i)(v)
\end{aligned}
$$

Note that the $\sigma$-operator presupposes the existence of a maximal sum individual. This means that the $\sigma$-term in (5) is undefined if $P$ has an empty extension at the index assigned to $i$. Now consider in (6) the translation of the focused $w h$-pronoun $w h o_{n}{ }^{\mathrm{Fe}}$ (where $\alpha^{\prime}$ is the translation of $\alpha$ ).

$$
\begin{aligned}
\text { who }_{n}{ }^{\mathrm{Fe}} \leadsto & \mathrm{F}_{\mathrm{e}}{ }^{\prime}\left(\lambda i . \mathrm{who}_{n}{ }^{\prime}\right) \\
& =\left[\lambda \mathscr{Q} \lambda P . \mathscr{Q}(i)\left(\lambda i \lambda v^{\prime}\left(v^{\prime}=\sigma v \cdot P(i)(v)\right)\right)\right]\left(\lambda i \lambda P . \exists u_{n} . P(i)\left(u_{n}\right)\right) \\
& =\lambda P . \exists u_{n}\left(u_{n}=\sigma v \cdot P(i)(v)\right)
\end{aligned}
$$

Due to the $\sigma$-operator, the generalized quantifier derived in (6) does not give a defined result if it applies to a property that has an empty extension at the index assigned to $i$. This will later be shown to be the source of a number of answerhood conditions and to lead to intervention effects in wh-questions.

\subsection{Question Words are Dynamic Binders}

Van Rooy (1998) observes that question words can serve as antecedents for anaphoric pronouns. This is illustrated in (7) with an example of discourse anaphora.

\footnotetext{
${ }^{9}$ For a proper definition, see Haida (2007).

${ }^{10}$ According to Link (1983), the $\sigma$-operator gives the maximal sum individual that satisfies the predicate it applies to. One reason to assume instead a definition along the lines of (5) is that $w h$-pronouns without a plural form can have a plural interpretation. See section 4.3 for relevant discussion.
} 
(7) Who $\mathrm{W}_{i}$ won the women's high jump? What hight did she jump? $_{i}$

We also find cases of donkey anaphora, that is, anaphoric binding in the scope of an operator that lends universal force to the anaphoric binder. The double question in (8) provides an example of this type of anaphora.

(8) Wer kaufte was $_{i}$ und verschenkte $e_{i}$ sofort? $^{2}$

(German) who bought what and gave away it immediately

literally: 'Who bought what ${ }_{i}$ and gave $\mathrm{it}_{i}$ away immediately?'

To see that (8) indeed exhibits discourse anaphora, note that in-situ wh-phrases do not undergo covert phrasal movement in German (see Pesetsky 2000, Beck 2006). ${ }^{11}$ Hence, we can conclude that the pronoun es is not syntactically bound by the insitu wh-phrase was. ${ }^{12}$ Hence, es cannot receive a bound-variable interpretation. Furthermore, note that (8) can be given the pair-list answer in (9).

(9) MARIA kaufte [einen $\mathrm{SCHAL}_{i}$ und verschenkte ihn ${ }_{i}$ sofort, PAUL kaufte BLUMEN B $_{j}$ und verschenkte sie $_{j}$ sofort, ...

'MARIA bought [a SCARF $]_{i}$ and gave it $_{i}$ away immediately, PAUL bought FLOWERS ${ }_{j}$ and gave them ${ }_{j}$ away immediately,...'

This shows that the anphoric binder was and the anaphoric pronoun es are in the scope of the same question-formation operator. Below we will see that this operator (denoted by the interrogative complementizer) lends universal force to the question words in its scope.

I take the above observations to show that question words denote dynamic existential quantifiers. Another reason for this assumption is that it leads to a variant of the partition theory of questions (Groenendijk and Stokhof 1982) that allows us to integrate the semantic assumptions of the two previous sections into a coherent theory. The dynamic-semantic variant of the partition theory and its properties are discussed in the remainder of this paper.

\section{A Dynamic-Semantic Variant of the Partition Theory of Questions}

\subsection{A Preview of the Translation of Wh-Questions}

I assume that in wh-ex-situ languages, simple $w h$-questions have an LF structure as exemplified in (10) for the the question "Who called?".

(10) $\left[\mathrm{CP}^{\mathrm{C}} \mathrm{C}^{[+\mathrm{Q}]}\left[\mathrm{FocP}_{\mathrm{Fho}} \mathrm{who}_{1}{ }^{\mathrm{Fe}}\left[\mathrm{TP} t_{1}\right.\right.\right.$ called $\left.\left.]\right]\right]$

\footnotetext{
${ }^{11}$ For a discussion of relevant data, see section 5 .

${ }^{12}$ I assume that (8) has the LF structure sketched in (i).

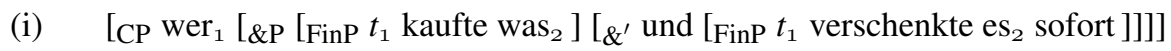

In (i), the ex-situ wh-phrase wer moves across-the-board from a coordinated structure of two FinPs (for this category, see Rizzi 1997).
} 
For reasons of space, I will not discuss the syntactic assumptions that go into deriving (10). Let me simply point out that the interrogative complementizer $\mathrm{C}^{[+\mathrm{Q}]}$ c-commands the $w h$-phrase even after it underwent movement into the left periphery of the clause. This movement is triggered by the $w h$-feature and the $\mathrm{F}_{\mathrm{e}}$ feature of the $w h$-phrase (in combination with properties of the left-peripheral heads). ${ }^{13}$

I assume that $\mathrm{C}^{[+\mathrm{Q}]}$ denotes an operator defining a partition of the set of indices. I. e., $\mathrm{C}^{[+\mathrm{Q}]}$ is translated as follows (cf. Groenendijk and Stokhof 1982).

$$
\mathrm{C}^{[+\mathrm{Q}]} \leadsto \lambda p \lambda j(p(i) \leftrightarrow p(j))
$$

This means that the LF structure in (10) translates into the term derived in (12) (where a trace $t_{n}$ translates into the variable $v_{n}$ and a constituent $\left[\alpha_{n} \beta\right]$ translates into $\left.\alpha_{n}{ }^{\prime}\left(\lambda i \lambda v_{n} \cdot \beta^{\prime}\right)\right){ }^{14}$

$$
\begin{aligned}
(10) \leadsto & \mathrm{C}^{[+\mathrm{Q}]^{\prime}}\left(\lambda i\left(\left[\mathrm{~F}_{\mathrm{e}}{ }^{\prime}\left(\lambda i \cdot \mathrm{who}_{1}{ }^{\prime}\right)\right]\left(\lambda i \lambda v_{1} \cdot \text { call }^{\prime}(i)\left(v_{1}\right)\right)\right)\right) \\
& =\lambda j\left(\exists u_{1}\left(u_{1}=\sigma v \cdot \underline{\text { call }}^{\prime}(i)(v)\right) \leftrightarrow \exists u_{1}\left(u_{1}=\sigma v \cdot \underline{\text { call }}^{\prime}(j)(v)\right)\right)
\end{aligned}
$$

In classical type logic, the $\lambda$-term derived in (12) does not represent the extension of a wh-question, which rather must be represented by a term of the following form (modulo logical equivalence).

$$
\lambda j\left(\lambda x_{1} \ldots \lambda x_{n} . \Phi=\lambda x_{1} \ldots \lambda x_{n} . \Psi\right)
$$

However, it can be shown that there is a denotational equivalence between (13) and the term in (14), ${ }^{15}$ namely if the existential quantifier and the biconditional operator are interpreted as dynamic operators (see Haida 2005, 2007). ${ }^{16}$

$$
\lambda j\left(\exists x_{1} \ldots \exists x_{n} . \neg \neg \Phi \leftrightarrow \exists x_{1} \ldots \exists x_{n} . \neg \neg \Psi\right)
$$

In a nutshell, the reason is this: The meaning of dynamic-logic formulas consists in their context change potential (Groenendijk and Stokhof 1991). The context change brought about by an existential formula $\exists x . P(x)$ affects the value of $x$ in subsequent formulas, namely in the following way. If an assignment for $x$ validates $P(x)$, this assignment persists as a possible value of $x$ in subsequent free occurrences of this variable. Hence, what constitutes the context change potential of $\exists x . P(x)$ is the totality of assignments for $x$ that validate $P(x)$. This means that the following equivalence holds (where $\simeq$ is the dynamic equivalence notion):

$$
\begin{aligned}
\exists x \cdot P(x) & \simeq \exists x \cdot Q(x) \\
& \text { iff } \\
\lambda x \cdot P(x) & =\lambda x \cdot Q(x)
\end{aligned}
$$

Therefore, (14) is denotationally equivalent to (13) if ' $\exists$ ' is the dynamic existential quantifer and ' $\leftrightarrow$ ' is the object-language correlate of ' $\simeq$ '.

\footnotetext{
${ }^{13}$ Remember that there is strong typological evidence that question words must be focused and, more specifically, that they bear the feature $\mathrm{F}_{\mathrm{e}}$ (for a systematic exception, see section 4.2). Therefore, it is reasonable to assume that ex-situ question words are focused even though there is only indirect phonological evidence for this. See Haida (2007) for a thorough discussion.

${ }^{14}$ The underlining of the translation of the verb will be explained in section 3.3.2.

${ }^{15}$ The double negation in (14) serves to hide the context change potential of $\Phi$ and $\Psi$.

${ }^{16}$ Groenendijk and Stokhof (1992: 122) already note that "it makes no difference whether we deal with $w h$-terms as a form of restricted $\lambda$-abstraction, or as dynamic existential quantification."
} 


\subsection{The Dynamic Type Logic MTy 3}

\subsubsection{Introduction}

In the following sections, I will provide the specifics of a dynamic logic that allows us to model the derivation of $w h$-question denotations. I will adopt one of the existing approaches, Muskens (1996), and adjust it to our needs. With reference to DRT, ${ }^{17}$ Muskens shows that dynamic predicate logic can be reduced to classical type theory, that is, he demonstrates that it is possible to represent the dynamics of DRT in terms of ordinary type logic. Against the background of this fact, Muskens convincingly argues that DRT expressions are best treated as abbreviations for typelogical expressions. This way, Montague Semantics and Discourse Representation are combined into a formalism that is both easy to use and mathematically rigorous. Below, I provide an implementation of this idea which follows Muskens' proposal in all respects except for the syntax of the dynamic-logic constructs: I will use the usual formula notation instead of Muskens' linearized DRT notation. The implementation is achieved (i) by specifying the type logic that serves as the target language into which the abbreviations are translated and (ii) by specifying how to translate the abbreviations. The reduction of dynamic predicate logic to classical type theory requires specific properties of the targeted type logic. The stipulations in the following two subsections ensure that the type system and the semantic models used for interpretation are suitable for our purposes.

\subsubsection{The Type System}

First, we need a type-logical correlate of what is referred to as context in dynamic predicate logic. Let us therefore assume that our type logic has a primitive type for contexts. Beyond this, we need primitive types for entities and possible-world indices. Thus, we assume three primitive types beyond $t$ : the type of entities $e$, the type of indices $s$, and the type of contexts $c .{ }^{18}$ Accordingly, we take Type, the set of types of our logic, to be the smallest set $Y$ such that (i) $e, s, c, t \in Y$, (ii) whenever $a, b \in Y,\langle a, b\rangle \in Y$.

As usual, the set of types is correlated with a family of sets of possible denotations. To be specific, let $\mathscr{D}, \mathscr{S}$, and $\mathscr{C}$ be pairwise disjoint, non-empty sets, which we regard as the set of entities, the set of indices, and the set of contexts, respectively. Then $\mathscr{D}_{a}$, the set of possible denotations of type $a$, is defined over $\mathscr{D}$, $\mathscr{S}$, and $\mathscr{C}$ in the following way: $\mathscr{D}_{e}=\mathscr{D}, \mathscr{D}_{s}=\mathscr{S}, \mathscr{D}_{c}=\mathscr{C}, \mathscr{D}_{t}=\{0,1\}$, and $\mathscr{D}_{\langle a, b\rangle}=\mathscr{D}_{b} \mathscr{D}_{a}$.

As will be explained shortly, contexts come into play in connection with semantic objects called registers. Registers are functions from contexts to entities,

\footnotetext{
${ }^{17}$ See Kamp and Reyle (1993).

${ }^{18}$ In Muskens (1996), contexts are called states and assigned the type $s$. However, this clashes with the usual convention to designate by $s$ the type of indices. Since we need both, indices and contexts, we use $s$ for indices and $c$ for contexts.
} 
that is, functions in $\mathscr{D}_{\langle c, e\rangle} \cdot{ }^{19}$ To characterize the purpose of these functions, let me simply point out that register constants will later be called discourse referents.

The following table gives an overview of the symbols that will be used to designate contexts and registers in the subsequent discussion.

\begin{tabular}{l|l|l} 
& contexts & registers \\
\hline type & $c$ & $\langle c, e\rangle$ \\
variables & $k, k^{\prime}, k_{1}, k_{2}, \ldots$ & $v, v^{\prime}, v_{1}, v_{2}, \ldots$ \\
$\begin{array}{l}\text { constants } \\
\begin{array}{l}\text { metavariables for } \\
\text { semantic objects } \\
\text { metavariables for } \\
\text { terms }\end{array}\end{array}$ & $\kappa, \kappa^{\prime}$ & $u, u_{1}, u_{2}, \ldots$ \\
& $k, k^{\prime}$ & $\rho, \rho_{1}, \rho_{2}, \ldots$ \\
& & $\delta, \delta_{1}, \delta_{2}, \ldots$
\end{tabular}

Henceforth, we speak of the value of a register $\rho$ in a context $\kappa$ to refer to the entity $\rho(\kappa)$. Furthermore, we speak of updating a register $\rho$ to a value $d$ when we mean selecting a context $\kappa$ such that $\rho(\kappa)=d$.

\subsubsection{The Structure of the Semantic Models}

For registers to serve their intended purpose, we need to impose a certain structure on the semantic models we use for interpretation. To this end, we have to refer to a specific subset of the set of registers $\mathscr{D}_{\langle c, e\rangle}$, namely to those registers denoted by a constant. Let us call these registers named registers, and let NMD be a predicate of type $\langle\langle c, e\rangle, t\rangle$ that singles them out. ${ }^{20}$ We want to ensure that each named register can be updated to any possible value without affecting the value of any other named register. To be able to formulate this in a concise way, let us define an abbreviation: ${ }^{21}$ For all terms $k$ and $k^{\prime}$ of type $c$ and all terms $\delta_{1}, \ldots, \delta_{n}$ of type $\langle c, e\rangle$, we write

$$
k\left[\delta_{1}, \ldots, \delta_{n}\right] k^{\prime} \quad \text { for } \quad \forall v\left(\left(\operatorname{NMD}(v) \wedge \delta_{1} \neq v \wedge \ldots \wedge \delta_{n} \neq v\right) \rightarrow v(k)=v\left(k^{\prime}\right)\right)
$$

According to this definition, a formula $k[\delta] k^{\prime}$ expresses that the contexts denoted by $k$ and $k^{\prime}$ differ at most in the value of the register denoted by $\delta$ whereby only

\footnotetext{
${ }^{19}$ In Muskens (1996), registers are atomic objects denoted by terms of another primitive type, $\pi$. Terms of type $\pi$ are related to terms of type $\langle c, e\rangle$ by means of a fixed non-logical constant $\mathrm{V}$ of type $\langle\pi,\langle c, e\rangle\rangle$. Thereby, $\mathrm{v}(u)(k)$ is taken to be the value of register $u$ in context $k$. I deviate from these assumptions to spare the additional type $\pi$. This does not complicate Muskens' approach, rather the contrary.

${ }^{20}$ This means that for all $\mathscr{M}$ and all $g$, the denotation of NMD must be as follows: $\forall \rho \in \mathscr{D}\langle c, e\rangle$ : $\llbracket \mathrm{NMD} \rrbracket \mathscr{M}_{g}(\rho)=1 \Leftrightarrow \rho \in \operatorname{ran}(F)$, where $F$ is the interpretation function of $\mathscr{M}$. The set of named registers corresponds to the range of the function denoted by $v$ in Muskens' original system. See fn. 19.

${ }^{21}$ Cf. Muskens (1996), p. 156.
} 
named registers are considered. (For reasons of brevity, I will henceforth no longer mention the restriction to named registers when referring to the above relation between contexts.) Now we can impose the following axiom on our semantic models to enable the abovementioned register updates. ${ }^{22}$

Axiom $1 \forall k \forall v \forall x\left(\operatorname{NMD}(v) \rightarrow \exists k^{\prime}\left(k[v] k^{\prime} \wedge v\left(k^{\prime}\right)=x\right)\right)$

Axiom 1 demands that for each context, each named register, and each entity, there is a second context that is just like the first one, except that the value of the given register is the given entity. To see the effect of axiom 1 on the structure of the affected domains, let us consider a model satisfying this axiom: Assume for simplicity that there are only three named registers, $\rho_{1}, \rho_{2}$, and $\rho_{3} \cdot{ }^{23}$ Then axiom 1 guarantees that for each triple $\left\langle d_{1}, d_{2}, d_{3}\right\rangle$ of elements of $\mathscr{D}_{e}$, there is a context $\kappa$ such that $\left\langle\rho_{1}(\kappa), \rho_{2}(\kappa), \rho_{3}(\kappa)\right\rangle=\left\langle d_{1}, d_{2}, d_{3}\right\rangle$. This is illustrated in table 1 below for $\mathscr{D}_{e}=\{0, \bullet\}$. In table 1 , the element in the $n$th row and $m$ th column is the value of $\rho_{m}$ in $\kappa_{n}$ (that is, $\rho_{m}\left(\kappa_{n}\right)$ ).

Table 1

\begin{tabular}{|c||c|c|c|}
\hline & $\rho_{1}$ & $\rho_{2}$ & $\rho_{3}$ \\
\hline \hline$\kappa_{1}$ & $\circ$ & $\circ$ & $\circ$ \\
\hline$\kappa_{2}$ & $\circ$ & $\circ$ & $\bullet$ \\
\hline$\kappa_{3}$ & $\circ$ & $\bullet$ & $\circ$ \\
\hline$\kappa_{4}$ & $\circ$ & $\bullet$ & $\bullet$ \\
\hline$\kappa_{5}$ & $\bullet$ & $\circ$ & $\circ$ \\
\hline$\kappa_{6}$ & $\bullet$ & $\circ$ & $\bullet$ \\
\hline$\kappa_{7}$ & $\bullet$ & $\bullet$ & $\circ$ \\
\hline$\kappa_{8}$ & $\bullet$ & $\bullet$ & $\bullet$ \\
\hline
\end{tabular}

Table 2

\begin{tabular}{|l||c|c|c|}
\hline & $x_{1}$ & $x_{2}$ & $x_{3}$ \\
\hline \hline$g_{1}$ & $\circ$ & $\circ$ & $\circ$ \\
\hline$g_{2}$ & $\circ$ & $\circ$ & $\bullet$ \\
\hline$g_{3}$ & $\circ$ & $\bullet$ & $\circ$ \\
\hline$g_{4}$ & $\circ$ & $\bullet$ & $\bullet$ \\
\hline$g_{5}$ & $\bullet$ & $\circ$ & $\circ$ \\
\hline$g_{6}$ & $\bullet$ & $\circ$ & $\bullet$ \\
\hline$g_{7}$ & $\bullet$ & $\bullet$ & $\circ$ \\
\hline$g_{8}$ & $\bullet$ & $\bullet$ & $\bullet$ \\
\hline
\end{tabular}

Now compare table 1 with table 2 . The rows of table 2 are the graphs of all assignment functions on the restricted domain $\left\{x_{1}, x_{2}, x_{3}\right\}$ (where $x_{1}, x_{2}$, and $x_{3}$ are variables of type $e$ ). That is, the element in the $n$th row and $m$ th column of table 2 is the value of $g_{n}$ applied to $x_{m}$. The correspondence between table 1 and table 2 shows that contexts can be considered as model-theoretic counterparts of assignment functions (if we disregard the reversal of the functor-argument relation with respect to registers and variables, respectively).

A second axiom demands that all constants of type $\langle c, e\rangle$ denote different registers. Let us call register-denoting constants discourse referents. Then the following axiom guarantees that an update on a discourse referent does not affect any other discourse referent. ${ }^{24}$

\footnotetext{
${ }^{22}$ Cf. AX1 on p. 156 in Muskens (1996). Note that due to the differences mentioned in fn. 19, we cannot simply adopt AX1. A further difference arises from the fact that Muskens distinguishes between two kinds of registers: for indefinites and for proper names. We will not use the latter kind of register, and hence disregard this distinction.

${ }^{23}$ Note, however, that this assumption is incompatible with axiom 2 introduced below.

${ }^{24} \mathrm{Cf}$. AX3 on p. 156 in Muskens (1996). Note, however, that AX3 should read " $\mathrm{v}\left(u_{n}\right) \neq \mathrm{v}\left(u_{m}\right)$ for each two different unspecific referents $u_{n}$ and $u_{m}$."
} 
Axiom $2 u_{n} \neq u_{m}$ for each two different discourse referents $u_{n}$ and $u_{m}$.

We will refer to the type logic specified above as $M T y_{3}\left(\right.$ Muskens' $\left.\mathrm{Ty}_{3}\right)$. This is in allusion to the fact that we are dealing with a three-sorted type logic and that we only consider models that satisfy Muskens' axioms 1 and 2.

\subsection{Translating Dynamic Predicate Logic into MTy 3}

\subsubsection{The Available Expressive Means}

To demonstrate what has been achieved so far, I will now show how to represent in $\mathrm{MTy}_{3}$ the context change potential of the existential formula $\exists x$. walk' $(x)$.

Assume that $u$ is a discourse referent (a constant of type $\langle c, e\rangle$ ) and that $k$ is a context variable. Then the $\lambda$-abstract in (15) is a meaningful expression of $\mathrm{MTy}_{3}$.

$$
\lambda k \cdot \operatorname{walk}^{\prime}(u(k))
$$

On the assumption that $u$ denotes the register $\rho$, (15) denotes (the characteristic function of) the set of all contexts $\kappa$ such that $\rho(\kappa)$ is an individual that walks. Furthermore, axiom 1 guarantees that for each individual $d$, there is a context $\kappa$ such that $\rho(\kappa)=d$ (remember that $\rho$ is a named register, $\rho$ being the denotation of $u$ ). Consequently, it holds that for each individual $d$ such that $d$ walks, there is a context $\kappa$ in the extension of (15) such that $\rho(\kappa)=d$. This means that the extension of (15) corresponds to the set $S$ of satisfying assignments of the open formula walk $(x): S$ is the set of all assignment functions $g$ such that $g(x)$ is an individual that walks; and for each individual $d$ such that $d$ walks, $S$ contains an assignment function $g$ such that $g(x)=d$.

The correspondence pointed out above shows that context variables can be considered as object-language correlates of assignment functions and can hence be used to represent the context change potential of formulas of dynamic predicate logic. In dynamic predicate logic, the context change potential of an existential formula $\exists x$.walk $(x)$ can be identified with its set of result contexts relative to an assignment $g$, the set $S_{g}$ (for simplicity, I omit reference to the model). An assignment $g^{\prime}$ is an element of $S_{g}$ iff (i) $g$ and $g^{\prime}$ differ at most in the value assigned to $x$ and (ii) $g^{\prime}(x)$ is an individual that walks. Furthermore, for each assignment function $g$ and each individual $d$ such that $d$ walks, there is an assignment function $g^{\prime} \in S_{g}$ such that $g^{\prime}(x)=d$.

Now consider the $\mathrm{MTy}_{3}$ term in (16), which will be shown to represent the context change potential characterized by $S_{g}$.

$$
\lambda k \lambda k^{\prime}\left(k[u] k^{\prime} \wedge \operatorname{walk}^{\prime}\left(u\left(k^{\prime}\right)\right)\right)
$$

The relation denoted by (16) holds between a pair of contexts $\kappa$ and $\kappa^{\prime}$ iff the following two conditions are met (where again we assume that $u$ denotes $\rho$ ): (i) $\kappa$ and $\kappa^{\prime}$ differ at most in the value of $\rho$ and (ii) $\rho\left(\kappa^{\prime}\right)$ is an individual that walks. Furthermore, we can derive from axiom 1 that for each context $\kappa$ and each individual $d$ such that $d$ walks, there is a context $\kappa^{\prime}$ such that $\left\langle\kappa, \kappa^{\prime}\right\rangle$ is in the extension of (16) and $\rho\left(\kappa^{\prime}\right)=d$. 
This shows that (16) represents the context change potential of the existential formula $\exists x$.walk $(x)$ (if, as we have done, we associate $u$ with $x$ ).

\subsubsection{The Abbreviations}

To highlight the relationship pointed out above, let us refer to the term in (16) and, generally, to terms of type $\langle c,\langle c, t\rangle\rangle$ as dynamic formulas. I will now define some abbreviations that allow us to designate dynamic formulas in a manner that shows their relation to corresponding formulas of dynamic predicate logic. ${ }^{25}$

First, we need a simple way of designating dynamic formulas that correspond to atomic formulas of dynamic predicate logic. Therefore, let us agree on the following: If $R$ is a constant of type $\left\langle s, e^{n} t\right\rangle$ (where $e^{0} t=t$ and $e^{k+1} t=\left\langle e, e^{k} t\right\rangle$ ), $i$ is a term of type $s$, and $\delta_{1}, \ldots, \delta_{n}$ are terms of type $\langle c, e\rangle$, we write

Abbr. $1 \underline{R}(i)\left(\delta_{1}, \ldots, \delta_{n}\right)$ for $\lambda k \lambda k^{\prime}\left(k=k^{\prime} \wedge R(i)\left(\delta_{1}(k), \ldots, \delta_{n}(k)\right)\right)$.

This abbreviation convention is illustrated in 17 (where $u$ is a discourse referent and $v$ and $v^{\prime}$ are register variables).
a. $\underline{\text { walk }}^{\prime}(i)(u) \quad$ is short for $\lambda k \lambda k^{\prime}\left(k=k^{\prime} \wedge\right.$ walk $\left.^{\prime}(i)(u(k))\right)$
b. $\quad \underline{\operatorname{love}^{\prime}}(i)\left(v, v^{\prime}\right)$ is short for $\lambda k \lambda k^{\prime}\left(k=k^{\prime} \wedge \operatorname{love}^{\prime}(i)\left(v(k), v^{\prime}(k)\right)\right)$

To designate dynamic formulas that correspond to complex formulas of dynamic predicate logic, we agree on the following: If $\Phi$ and $\Psi$ are dynamic formulas and $u$ is a discourse referent, ${ }^{26}$ we write
Abbr. $2 \neg \Phi$
for $\lambda k \lambda k^{\prime}\left(k=k^{\prime} \wedge \neg \exists k_{2} . \Phi(k)\left(k_{2}\right)\right)$,
$(\Phi \vee \Psi) \quad$ for $\quad \lambda k \lambda k^{\prime}\left(k=k^{\prime} \wedge \exists k_{2}\left(\Phi(k)\left(k_{2}\right) \vee \Psi(k)\left(k_{2}\right)\right)\right)$,
$(\Phi \rightarrow \Psi) \quad$ for $\quad \lambda k \lambda k^{\prime}\left(k=k^{\prime} \wedge \forall k_{2}\left(\Phi(k)\left(k_{2}\right) \rightarrow \exists k_{3} . \Psi\left(k_{2}\right)\left(k_{3}\right)\right)\right)$,
$(\Phi \leftrightarrow \Psi) \quad$ for $\quad \lambda k \lambda k^{\prime}\left(k=k^{\prime} \wedge \forall k_{2}\left(\Phi(k)\left(k_{2}\right) \leftrightarrow \Psi(k)\left(k_{2}\right)\right)\right)$,
$\forall u \Phi \quad$ for $\quad \lambda k \lambda k^{\prime}\left(k=k^{\prime} \wedge \forall k_{2}\left(k[u] k_{2} \rightarrow \exists k_{3} . \Phi\left(k_{2}\right)\left(k_{3}\right)\right)\right)$,

Abbr. $3 \quad \exists u \Phi \quad$ for $\lambda k \lambda k^{\prime} . \exists k_{2}\left(k[u] k_{2} \wedge \Phi\left(k_{2}\right)\left(k^{\prime}\right)\right)$,

$(\Phi \wedge \Psi) \quad$ for $\quad \lambda k \lambda k^{\prime} . \exists k_{2}\left(\Phi(k)\left(k_{2}\right) \wedge \Psi\left(k_{2}\right)\left(k^{\prime}\right)\right)$.

The above list is organized in such a way that Abbr. 2 subsumes the externally static

\footnotetext{
${ }^{25}$ Cf. Muskens (1996), p. 157. Muskens uses linearized DRS boxes to abbreviate dynamic formulas. This way, no confusion can arise by mistaking the abbreviations for ordinary formulas. On the other hand, it is (at least for me) somewhat difficult to grasp the meaning of these expressions. The format I propose allows to read the abbreviations as if they were DPL formulas (Groenendijk and Stokhof 1991).

${ }^{26}$ At first sight, an expression of the form $\exists u \Phi$ appears to express something nonsensical, namely quantification over a register constant. However, notice that the quantification is over context variables in the underlying $\mathrm{MTy}_{3}$ expression. Therefore, $\exists u \Phi$ is best pronounced as "there is an update of the register denoted by $u$ that satisfies $\Phi$." The same comments apply to the abbreviation $\forall u \Phi$, which is best pronounced as "every update of the register denoted by $u$ satisfies $\Phi . "$
} 
operators, ${ }^{27}$ and Abbr. 3 the externally dynamic ones. Note that the above conventions are direct translations of how the sematic value of the corresponding DPL formulas is defined. ${ }^{28}$ Therefore, we can be sure that, for instance, the dynamic formula abbreviated by $(\Phi \wedge \Psi)$ is in fact the dynamic conjunction of $\Phi$ and $\Psi$.

If no confusion can arise, we will speak of the abbreviations conforming to Abbr. 1-3 as of actual expressions of our logic.

\subsection{Some Logical Properties of Dynamic Formulas}

Dynamic formulas denote functions from contexts to (characteristic functions of) sets of contexts. In other words, dynamic formulas denote their context change potential. The logical notions of truth, validity and equivalence must be defined accordingly. Specifically, the notion of truth of a dynamic formula $\Phi$ must be defined relative to a context $\kappa:{ }^{29}$

\section{Definition 1 (Truth)}

$\Phi$ is true with respect to $g$ and $\kappa$ in $\mathscr{M} \Leftrightarrow \quad \exists \kappa^{\prime}: \llbracket \Phi \rrbracket \mathscr{M}, g(\kappa)\left(\kappa^{\prime}\right)=1$.

The notion of validity is defined as truth with respect to all models, assignments, and contexts:

\section{Definition 2 (Validity)}

$\Phi$ is valid $\Leftrightarrow \forall \mathscr{M} \forall g \forall \kappa: \Phi$ is true with respect to $g$ and $\kappa$ in $\mathscr{M}$.

Since dynamic formulas denote their context change potential, the notion of logical equivalence can be defined by sole reference to their extension:

Definition 3 (Equivalence) $\Phi \simeq \Psi \Leftrightarrow \forall \mathscr{M} \forall g: \llbracket \Phi \rrbracket_{\mathscr{M}, g}=\llbracket \Psi \rrbracket \mathscr{M}, g$.

Then we find that ' $\leftrightarrow$ ' is the object-language correlate of ' $\simeq$ ':

Fact $1 \Phi \simeq \Psi \Leftrightarrow \Phi \leftrightarrow \Psi$ is valid.

\subsection{The Translation of Wh-Questions}

By following Muskens in defining our dynamic-logic constructs, we avoided leaving the realm of ordinary type logic. Therefore, we now have at our disposal the full power of $\lambda$-abstraction. The translations of the relevant lexical items (and the rules of composition) have already been presented in the previous sections. However, at the point they were introduced, the semantic type of these translations could not be clearly stated. This is made up for in the following table. Below, we abbreviate

\footnotetext{
${ }^{27}$ For simplicity, we assume that the biconditional operator is externally static. See section 2.3 for a problematization of this assumption.

${ }^{28}$ See definition 2 in Groenendijk and Stokhof (1991). This holds with the exception of the biconditional operator, which, as already mentioned, is not defined in Groenendijk and Stokhof (1991).

${ }^{29}$ Cf. Muskens (1996), p. 172.
} 
$\langle c,\langle c, t\rangle\rangle$ as $\underline{t}$ and $\langle c, e\rangle$ as $\underline{e}$. That is, we say that dynamic formulas are of type $\underline{t}$ and that discourse referents and register terms are of type $\underline{e}$. With these notational conventions, we highlight that the $\mathrm{MTy}_{3}$ translations are isomorphic to their (nondynamic) $\mathrm{Ty}_{2}$ counterparts.

\section{$\mathrm{MTy}_{3}$ translations of some lexical items (mostly repeated from above)}

\begin{tabular}{lll} 
LI & \multicolumn{1}{l}{ Translation } & Type \\
$\mathrm{C}^{[+\mathrm{Q}]}$ & $\leadsto \lambda p \lambda j(p(i) \leftrightarrow p(j))$ & $\langle\langle s, \underline{t}\rangle,\langle s, \underline{t}\rangle\rangle$ \\
$\mathrm{who}_{n} \leadsto \lambda P \cdot \exists u_{n} \cdot P(i)\left(u_{n}\right)$ & $\langle\langle s,\langle\underline{e}, \underline{t}\rangle\rangle, \underline{t}\rangle$ \\
$\mathrm{F}_{\mathrm{e}} \leadsto \lambda \mathscr{Q} \lambda P \cdot \mathscr{Q}(i)\left(\lambda i \lambda v^{\prime}\left(v^{\prime}=\sigma v \cdot P(i)(v)\right)\right)$ & $\langle\langle\langle s,\langle\underline{e}, \underline{t}\rangle\rangle, \underline{t}\rangle,\langle\langle s,\langle\underline{e}, \underline{t}\rangle\rangle, \underline{t}\rangle\rangle$ \\
call & $\leadsto \lambda v \cdot \underline{\operatorname{call}}^{\prime}(i)(v)$ & $\langle\underline{e}, \underline{t}\rangle$
\end{tabular}

\section{Accounting for Presuppositions of Wh-Questions}

There is a number of answerhood conditions that are not accounted for by the pertinent semantic theories of questions (for an overview and a descriptive account, see Dayal 2002). In the following sections, I will identify these answerhood conditions as presuppositions triggered by $\mathrm{F}_{\mathrm{e}}$ features of question words.

\subsection{The Existential Presupposition of Simple Wh-Questions}

It is often assumed that simple $w h$-questions have an existential presupposition: By asking "Who called?", the questioner presupposes that someone called (see, among many others, Keenan 1971). This assumption is sometimes challenged by pointing out that (18-A) is a coherent reply to the question in (18-Q).

(18) Q: Who called?

A: No one called.

However, the coherence of this reply does not show that it is a (semantic) answer to the question posed. It is well known that negations can be used to protest against a presupposition. Hence, (18-A) could be a protest against the existential presupposition of (18-Q). In my view, the inadequacy of the reply in (19) provides more conclusive evidence for judging the correctness of the assumption under discussion.

(19) A: \# Someone called.

If (18-Q) did not presuppose that someone called, the reply in (19-A) would be an informative answer, as it rules out the possibility that in fact no one called. However, (19-A) seems to be inadequate precisely because it is a completely uninformative reply. Therefore, I assume that simple $w h$-questions have indeed an existential presupposition.

This is predicted by the analysis presented above. As detailed in section 3.1, $(18-Q)$ has the LF structure in (20a), which is translated into the term in (20b). 


$$
\begin{aligned}
& \text { a. }\left[{ } _ { \mathrm { CP } } \mathrm { C } ^ { [ + \mathrm { Q } ] } { } _ { [ \mathrm { FocP } } \mathrm { who } _ { 1 } { } ^ { \mathrm { Fe } } \left[\mathrm{TP} t_{1}\right.\right. \text { called ]]] } \\
& \text { b. } \lambda j\left(\exists u_{1}\left(u_{1}=\sigma v \cdot \underline{\text { call }}^{\prime}(i)(v)\right) \leftrightarrow \exists u_{1}\left(u_{1}=\sigma v \cdot \text { call }^{\prime}(j)(v)\right)\right)
\end{aligned}
$$

Note that the $\sigma$-terms in (20b) are defined only if someone called at the respective index. Hence, the intension of (20b) only defines a partition of a subset of the set of indices, namely of those indices $i$ for which it holds that someone called at $i$. This means that (20b) captures the existential presupposition of (18-Q).

\subsection{The Pair-list Presupposition of Multiple Wh-Questions}

In the following paragraphs, I will argue that the pair-list (or rather tuple-list) presupposition of multiple $w h$-questions arises from the distribution of $\mathrm{F}_{\mathrm{e}}$ features in these questions.

In Hungarian, there is only one preverbal focus position. This is shown by the fact that a phrase preceding the immediately preverbal phrase, as for example the phrase János in (21), cannot receive a focal interpretation (cf. É. Kiss 1991).

(21) János Marit kisérte haza.

John.NOM Mary.ACC escorted home

a. * 'It was Mary that John ${ }_{\mathrm{Foc}}$ escorted home.'

b. 'As for John, it was Mary who he escorted home.'

Consequently, ${ }^{30}$ a $w h$-phrase cannot precede a preverbal phrase, as is shown by the unacceptability of (22) (cf. Suranyi 2002).

$$
\begin{aligned}
& \text { * Mit Marinak adott János? } \\
& \text { what.ACC Mary.DAT gave John.NOM } \\
& \text { intended: 'What did John give to Mary Foc?' }
\end{aligned}
$$

However, there is a systematic exception to this generalization: In multiple whquestions, more than one $w h$-phrase can appear preverbally. This is exemplified by the questions in (23) (see É. Kiss 1998: 263, n10).

$$
\begin{aligned}
& \text { a. Ki mit hozott Marinak? } \\
& \text { who what.ACC brought Mary.DAT }
\end{aligned}
$$

'Tell me about each person what he brought for Mary!'

b. Mit ki hozott Marinak?

'Tell me about each object who brought it for Mary!'

This suggests that the distribution of $F_{e}$ features in (23a) and (23b) is as given in the (simplified) LF structures in (24a) and (24b), respectively (where $\alpha$ is some projection in the left periphery, possibly TopP).

$$
\begin{aligned}
& \text { a. } \left.\left[{ }_{\mathrm{CP}} \mathrm{C}^{[+\mathrm{Q}]}\left[\alpha{ }_{\alpha} \mathrm{ki}_{1}\left[{ }_{\mathrm{FocP}} \mathrm{mit}_{2}{ }^{{ }_{\mathrm{Fe}}}{ }_{[\mathrm{TP}} t_{1} \text { hozott Marinak } t_{2}\right]\right]\right]\right] \\
& \text { b. } \left.\left[{ }_{\mathrm{CP}} \mathrm{C}^{[+\mathrm{Q}]}\left[\alpha{ }_{\alpha} \mathrm{mit}_{2}\left[{ }_{\mathrm{FocP}} \mathrm{ki}_{1}{ }^{{ }{ }^{2}}{ }_{[\mathrm{TP}} t_{1} \text { hozott Marinak } t_{2}\right]\right]\right]\right]
\end{aligned}
$$

\footnotetext{
${ }^{30}$ See section 2.2.
} 
Crucially, in both LF structures only one of the wh-pronouns bears an $\mathrm{F}_{\mathrm{e}}$ feature, namely the immediately preverbal one.

Now note that the questions in (23) differ in meaning although both ask for a list of buyers paired with the things they bought. It is reasonable to assume that the performative paraphrases given in (23a) and (b) reflect different pair-list presuppositions, the presuppositions in (25a) and (b), respectively.

a. $\dashv$ For every $x$, there is a $y$ such that $x$ brought $y$ for Mary.

b. $\dashv$ For every $y$, there is an $x$ such that $x$ brought $y$ for Mary.

I will now show that (25a) and (b) are presuppositions of the denotations of the LF structures in (24). The translation of (24a) and (b) is given in (26a) and (b), respectively (where mary is short for $\lambda k$.mary and mary is a constant of type $e$ ).

$$
\begin{aligned}
& \text { a. }(24 a) \leadsto \mathrm{C}^{[+\mathrm{Q}]^{\prime}}\left(\lambda i\left(\mathrm{ki}_{1}{ }^{\prime}\left(\lambda i \lambda v_{1}\left(\left[\mathrm{~F}_{\mathrm{e}}{ }^{\prime}\left(\mathrm{mit}_{2}{ }^{\prime}\right)\right]\left(\lambda i \lambda v_{2} \cdot \mathrm{TP}^{\prime}\right)\right)\right)\right)\right) \\
& =\mathrm{C}^{[+\mathrm{Q}]^{\prime}}\left(\lambda i \cdot \exists u_{1} \exists u_{2}\left(u_{2}=\sigma v \cdot \operatorname{bring}^{\prime}(i)\left(u_{1}, \operatorname{mary}, v\right)\right)\right) \\
& =\lambda j\left(\exists u_{1} \exists u_{2}\left(u_{2}=\sigma v \cdot \text { bring }^{\prime}(i)\left(u_{1}, \underline{\operatorname{mary}}, v\right)\right) \leftrightarrow\right.
\end{aligned}
$$

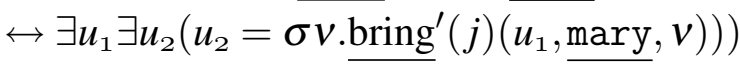

$$
\begin{aligned}
& \text { b. }(24 b) \leadsto \lambda j\left(\exists u_{1} \exists u_{2}\left(u_{1}=\sigma v \cdot \operatorname{bring}^{\prime}(i)\left(v, \operatorname{mary}, u_{2}\right)\right) \leftrightarrow\right. \\
& \leftrightarrow \exists u_{1} \exists u_{2}\left(u_{1}=\overline{\sigma v \cdot \operatorname{bring}^{\prime}}(j) \overline{\left.\left.\left(v, \underline{\text { mary }}, u_{2}\right)\right)\right)}\right.
\end{aligned}
$$

Consider the $\mathrm{MTy}_{3}$ term derived in (26a) (repeated in 27).

$$
\begin{gathered}
\lambda j\left(\exists u_{1} \exists u_{2}\left(u_{2}=\sigma v \cdot \underline{\operatorname{bring}^{\prime}}(i)\left(u_{1}, \underline{\operatorname{mary}}, v\right)\right) \leftrightarrow\right. \\
\leftrightarrow \exists u_{1} \exists u_{2}\left(u_{2}=\sigma v \cdot \underline{\operatorname{bring}^{\prime}}(j)\left(\underline{\left.\left.\left.u_{1}, \underline{\operatorname{mary}}, v\right)\right)\right)}\right.\right.
\end{gathered}
$$

Note that on both sides of the biconditional operator, the dynamic existential quantifier $\exists u_{1}$ binds an occurrence of $u_{1}$ within a $\sigma$-term. The dynamic biconditional operator requires that the formulas in the scope of $\exists u_{1}$ are equivalent for all valuations of $u_{1}$. Consequently, the biconditional in (27) is defined only if everybody brought something for Mary at the indices assigned to $i$ and $j$. Hence, the intension of (27) defines only a partition of a subset of the set of indices, namely of those indices $i$ for which it holds that everybody brought something for Mary at $i$. This means that (27) captures the pair-list presupposition of (23a).

In the same way, it can be shown that the $\mathrm{MTy}_{3}$ term in (26b) captures the pair-list presupposition of (23b), that is, the presupposition in (25b).

\subsection{Other Presuppositions of Wh-Questions}

Other presuppositions of simple and multiple $w h$-questions - e.g., the uniqueness presupposition of singular which-questions (see 28) - follow in the same way.

a. Which student called?

b. $\dashv$ One and only one student called.

For reasons of space, this cannot be shown in detail here. Let me simply mention that (28b) is a presupposition of (29b), which is the $\mathrm{MTy}_{3}$ translation of the LF structure in (29a) (for details, see Haida 2007). 

a. [CP $\mathrm{C}^{[+\mathrm{Q}]}\left[\mathrm{FocP}\right.$ [DP which ${ }_{1}{ }^{\mathrm{Fe}}$ student ] [TP [DP which ${ }_{1}{ }^{\mathrm{Fe}_{\mathrm{e}}}$ student ] called ]]]
b. $\mathrm{C}^{[+Q]^{\prime}}\left(\lambda i . \exists u_{1}\left(\underline{\operatorname{At}}\left(u_{1}\right) \wedge u_{1}=\sigma v\left(\underline{\text { student }}^{\prime}(i)(v) \wedge \underline{\text { call }}^{\prime}(i)(v)\right)\right)\right)$

To achieve this result, it is crucial that singular which-phrases of the form which $h_{n} \alpha$ receive the translation in (30).

$$
\operatorname{which}_{n} \alpha \leadsto \lambda P . \exists u_{n}\left(\underline{\operatorname{At}}\left(u_{n}\right) \wedge \alpha^{\prime}\left(u_{n}\right) \wedge P(i)\left(u_{n}\right)\right)
$$

Thereby, $\underline{\mathbf{A t}}\left(u_{n}\right)$ is the requirement that (the value of) $u_{n}$ is an atomic individual in the sense of Link (1983). ${ }^{31,32}$

\section{Accounting for Intervention Effects in Wh-Questions}

The deviance of the constructions in (31) exemplifies a class of phenomena that are known as intervention effects in wh-questions (Beck 1996, 2006).

a. ?? Wer hat nicht was gelesen?

(German) who has not what read intended: 'Who didn't read what?'

b. * Minsu-man nuku-lûl po-ass-ni?

(Korean) Minsu-only who-ACC see-PAST-Q intended: 'Who did only Minsu see?'

I adopt the assumption of Pesetsky (2000) that intervention effects can be obviated by covert phrasal movement. Then the deviance of (31a) and (b) shows that in Korean and German, in-situ wh-phrases do not undergo covert phrasal movement. Thus, intervention effects arise in the following LF configuration (Beck 2006).

$$
*\left[\mathrm{C}^{[+\mathrm{Q}]}[\ldots[\text { intervener }[\ldots w h \text {-phrase... }]] \ldots]\right]
$$

In (32), $\mathrm{C}^{[+\mathrm{Q}]}$ is the licensing complementizer of the $w h$-phrase, and the intervener belongs to a class of expressions containing (counterparts of) the following elements: (i) focusing elements such as only, even, and also; (ii) quantificational elements such as (almost) every, most, always, often, and the sentence negation not.

There is already a number of semantic approaches that aim to explain intervention effects in $w h$-questions. However, none of these approaches is fully satisfactory. The focus-semantic approach of Beck (2006) struggles with some of the quantificational interveners and cannot account for the indefinite-interrogative

\footnotetext{
${ }^{31} \operatorname{At}(\delta)$ is short for $\lambda k \lambda k^{\prime}\left(k=k^{\prime} \wedge \partial \forall x(x \Pi \delta(k) \rightarrow x=\delta(k))\right)$.

${ }^{32}$ The contrast in (i) shows that $w h$-pronouns allow for a plural interpretation even though they are morphosyntactically singular.

a. I wonder who is talking to one another (in the room next door).

b. * I wonder which person is talking to one another (in the room next door).

Note that speakers of American English tend to judge (i-a) as unacceptable too. This suggests that for these speakers, reciprocals must have an antecedent that is morphosyntactically as well as semantically plural.
} 
affinity. The dynamic-semantic approaches of Honcoop (1996), Butler (2000) and Haida (2005) fail to account for the intervener status of focusing elements. Moreover, these approaches only predict the distribution of intervention effects and require an additional stipulation to account for the deviance of constructions of the form in (32) (see also Kratzer and Shimoyama 2002).

I will show in the following that the deviance of (31a) and (b) is straightforwardly accounted for by the analysis presented in this paper. First, consider in (33) the simplified LF structure of (31a) (represented using the English glosses).

$$
\left.\left.\left[\mathrm{CP} \mathrm{C}^{[+\mathrm{Q}]}\left[\mathrm{FocP}_{\mathrm{Who}} \mathrm{W}_{\nu \mathrm{P}} \operatorname{not}{ }_{{ }_{\nu \mathrm{P}}} t_{1}\left[\mathrm{vP} \mathrm{what}_{2}{ }^{\mathrm{F}_{\mathrm{e}}} \mathrm{read}\right]\right]\right]\right]\right]
$$

The translation of (33) is given in (34).

$$
\mathrm{C}^{[+\mathrm{Q}]^{\prime}}(\lambda i . \exists u_{1} \overbrace{\neg \exists u_{2}\left(u_{2}=\sigma v \cdot \underline{\operatorname{read}}^{\prime}(i)\left(u_{1}, v\right)\right)}^{\Phi})
$$

With respect to (34), we observe the following. Due to the presuppostion of the $\sigma$-operator, the dynamic formula $\Phi$ is false (in all contexts) if it is defined. Moreover, due to the negation, $\Phi$ does not have context change potential. That is, $\Phi$ is semantically inert. Hence, the intension of (34) defines the trivial partition of the subset of indicies on which it is defined. This means that (33) does not give rise to a proper semantic question, and this explains the deviance of (31a).

Now consider the LF structure of (31b), which is given in (35) (using the English glosses).

$$
\left[\mathrm{CP}^{[+\mathrm{Q}]}\left[\mathrm{TP}_{\mathrm{P}} \text { Minsu-only }{ }_{1}\left[\mathrm{vP}_{\mathrm{Pho}}{ }^{\mathrm{Fe}} \text { saw }\right]\right]\right]
$$

I assume that a DP $\alpha$ suffixed with -man 'only' receives the following translation. ${ }^{33}$

$$
\alpha \text {-only }_{n} \leadsto \lambda P\left(\forall u_{n}\left(P(i)\left(u_{n}\right) \rightarrow u_{n} \text { is } \alpha^{\prime}\right) \wedge \partial P(i)\left(\alpha^{\prime}\right)\right)
$$

Thereby, ' $\partial$ ' is (the dynamic version of) Beaver's partial operator (Beaver 2001). According to this assumption, (35) has the translation given in (37).

$$
\begin{aligned}
& \mathrm{C}^{[+\mathrm{Q}]^{\prime}}(\lambda i( \forall u_{1}\left(\exists u_{2}\left(u_{2}=\sigma v \cdot \underline{\operatorname{see}^{\prime}}(i)\left(u_{1}, v\right)\right) \rightarrow u_{1} \underline{\text { is }} \underline{\operatorname{minsu}}\right) \wedge \\
&\left.\left.\wedge \partial \exists u_{2}\left(u_{2}=\sigma v \cdot \underline{\operatorname{see}^{\prime}}(i)(\underline{\operatorname{minsu}}, v)\right)\right)\right)
\end{aligned}
$$

With respect to (37), we observe the following. Due to the $\sigma$-operator, the existential formula in the scope of the universal quantifier is true for all valuations of $u_{1}$ if it is defined. Hence, if there is an individual other than Minsu, the universal formula is false if it is defined. Thus, the conjunction in (37) does not have context change potential. This is trivially the case if, otherwise, Minsu is the only individual. Hence, the intension of (37) defines the trivial partition of the subset of indices on which it is defined. Thus, (35) does not give rise to a proper semantic question.

In Haida (2007), it is shown that this analysis extends to all of the quantificational interveners and to the focusing element even. However, it remains to be shown how to account for the intervener status of also.

\footnotetext{
${ }^{33} \delta$ is $\delta^{\prime}$ is short for $\lambda k \lambda k^{\prime}\left(k=k^{\prime} \wedge \delta(k)=\delta^{\prime}(k)\right)$.
} 


\section{Conclusion}

The analysis presented in this paper took as its starting point two typological tendencies and another, related empirical phenomenon. The phenomena considered were semantically analyzed in a straightforward manner and an integration into existing semantic frameworks could be achieved. In addition to this, it was shown that the proposed analysis explains a number of other significant properties of the constructions under consideration.

\section{References}

Beaver, David Ian: 2001, Presupposition and Assertion in Dynamic Semantics. CSLI Publications, Stanford, CA.

Beck, Sigrid: 1996, 'Quantified Structures as Barriers for LF Movement', Natural Language Semantics 4, 1-56.

Beck, Sigrid: 2006, 'Intervention Effects Follow from Focus Interpretation', Natural Language Semantics 14, 1-56.

Bhat, D. N. S.: 2000, 'The Indefinite-interrogative Puzzle', Linguistic Typology 4, 365-400.

Butler, Alastair: 2000, 'Semantic Control and Intervention', in C. Pilière (ed.), Proceedings of the ESSLLI-2000 Student Session, 45-54.

Choe, Hyon Sook: 1995, 'Focus and Topic Movement in Korean and Licensing', in K. É. Kiss (ed.), Discourse Configurational Languages, 269-334. Oxford University Press, New York.

Dayal, Veneeta: 2002, 'Single-pair vs. Multiple-pair Answers: Wh in-situ and Scope', Linguistic Inquiry 33, 512-520.

É. Kiss, Katalin: 1991, 'Logical Structure in Syntactic Structure', in C.-T. J. Huang and R. May (eds.), Logical Structure and Linguistic Structure, 111-147. Kluwer, Dordrecht.

É. Kiss, Katalin: 1995, 'Introduction', in K. É. Kiss (ed.), Discourse Configurational Languages, 3-27. Oxford University Press, New York.

É. Kiss, Katalin: 1998, 'Identificational Focus versus Information Focus', Language 74, 245-273.

Groenendijk, Jeroen and Martin Stokhof: 1982, 'Semantic Analysis of WhComplements', Linguistics and Philosophy 5, 175-233.

Groenendijk, Jeroen and Martin Stokhof: 1991, 'Dynamic Predicate Logic', Linguistics and Philosophy 14, 39-100.

Groenendijk, Jeroen and Martin Stokhof: 1992, 'A Note on Interrogatives and Adverbs of Quantification', in C. Barker and D. Dowty (eds.), Proceedings from the Second Conference on Semantics and Linguistic Theory, 99-124. The Ohio State University, Columbus, Ohio.

Haida, Andreas: 2005, 'Dynamic Wh-Terms', in P. Dekker and M. Franke 
(eds.), Proceedings of the Fifteenth Amsterdam Colloquium, 95-100. ILLC/Department of Philosophy, University of Amsterdam, Amsterdam.

Haida, Andreas: 2007, The Indefiniteness and Focusing of Wh-Words, Doctoral Dissertation, Humboldt-Universität, Berlin.

Haspelmath, Martin: 1997, Indefinite Pronouns. Clarendon, Oxford.

Honcoop, Martin: 1996, 'Towards a Dynamic Semantics Account of Weak Islands', in T. Galloway and J. Spence (eds.), Proceedings from Semantics and Linguistic Theory VI, 93-110. Cornell University, Ithaca.

Kamp, Hans and Uwe Reyle: 1993, From Discourse to Logic (Studies in Linguistics and Philosophy 42). Kluwer, Dordrecht.

Karttunen, Lauri: 1977, 'Syntax and Semantics of Questions', Linguistics and Philosophy 1, 3-44.

Keenan, Edward: 1971, 'Quantifier Structures in English', Foundations of Language 7, 225-284.

Kratzer, Angelika and Junko Shimoyama: 2002, 'Indeterminate Pronouns: The View from Japanese', in Y. Otsu (ed.), The Proceedings of the Third Tokyo Conference on Psycholinguistics, 1-25. Hituzi Syobo, Tokyo.

Link, Godehard: 1983, 'The Logical Analysis of Plurals and Mass Terms: A Lattice-theoretical Approach', in R. Bäuerle, C. Schwarze, and A. von Stechow (eds.), Meaning, Use, and Interpretations of Language, 302-323. Walter de Gruyter, Berlin.

Muskens, Reinhard: 1996, 'Combining Montague Semantics and Discourse Representation', Linguistics and Philosophy 19, 143-186.

Pesetsky, David: 2000, Phrasal Movement and Its Kin. MIT Press, Cambridge, Mass.

Rizzi, Luigi: 1997, 'The Fine Structure of the Left Periphery', in L. Haegeman (ed.), Elements of Grammar: Handbook in Generative Syntax, 281-337. Kluwer, Dordrecht.

Suranyi, László Balázs: 2002, 'Syntactic Mechanisms and Interpretation of WhSaturation in Multiple Wh-Dependencies'. ms., Universiteit Utrecht.

Szabolcsi, Anna: 1981, 'The Semantics of Topic-Focus Articulation', in G. Groenendijk, T. Janssen, and M. Stokhof (eds.), Formal Methods in Study of Language (Mathematical Centre Tracts 136), 513-541. Matematisch Centrum, Amsterdam.

Szabolcsi, Anna: 1994, 'All Quantifiers are not Equal: The Case of Focus', Acta Linguistica Hungarica 42, 171-187.

Van Rooy, Robert: 1998, 'Modal Subordination in Questions', in J. Hulstijn and A. Nijholt (eds.), Proceedings of TWENDIAL 98, 237-248. University of Twente, Enschede.

Van Valin, Robert D., Jr.: 1993, 'A Synopsis of Role and Reference Grammar', in R. Van Valin (ed.), Advances in Role and Reference Grammar, 1-164. John Benjamins, Amsterdam.

Zaefferer, Dietmar: 1991, 'Weiß wer was? Wer weiß was? Wer was weiß...wInterrogative und andere w-Konstruktionen im Deutschen', in M. Reis and I. Rosengren (eds.), Fragesätze und Fragen, 77-93. Niemeyer, Tübingen. 